Epidemiology and Infection

cambridge.org/hyg

\section{Original Paper}

Cite this article: Pogreba-Brown K, O'Connor P, Matthews J, Barrett E, Bell ML (2018). Case-case analysis of Campylobacter and Salmonella - using surveillance data for outbreak investigations and monitoring routine risk factors. Epidemiology and Infection 146, 1916-1921. https://doi.org/10.1017/ S0950268818002200

Received: 30 January 2018

Accepted: 17 July 2018

First published online: 10 August 2018

Key words:

Campylobacter; case-case; outbreaks; Salmonella; surveillance

Author for correspondence:

K. Pogreba-Brown, E-mail: kpogreba@email. arizona.edu

\title{
Case-case analysis of Campylobacter and Salmonella - using surveillance data for outbreak investigations and monitoring routine risk factors
}

\section{K. Pogreba-Brown ${ }^{1}$, P. O'Connor ${ }^{1}$, J. Matthews ${ }^{2}$, E. Barrett ${ }^{1}$ and M. L. Bell ${ }^{1}$}

\footnotetext{
${ }^{1}$ University of Arizona, Mel and Enid Zuckerman College of Public Health, Department of Epidemiology and Biostatistics, Tucson, Arizona, USA and ${ }^{2}$ Maricopa County Department of Public Health, Office of Epidemiology, Phoenix, Arizona, USA
}

\begin{abstract}
Utilising routine surveillance data, this study presents a method for generating a baseline comparison that can be used in future foodborne outbreak investigations following a case-case methodology. Salmonella and Campylobacter cases (2012-2015) from Maricopa County, $\mathrm{AZ}$ were compared to determine differences in risk factors, symptoms and demographics. For foods and other risk factors, adjusted odds ratios were developed using Campylobacter as the reference. Comparisons were also made for three major Salmonella subtypes, Typhimurium, Enteritidis and Poona as compared with Campylobacter. Salmonella cases were younger, while Campylobacter cases were more Hispanic and female. Campylobacter cases reported consuming peppers, sprouts, poultry, queso fresco, eggs and raw nuts more and reported contact with animal products, birds, visiting a farm or dairy, owning a pet, a sick pet, swimming in a river, lake or pond, or handling multiple raw meats more. Salmonella cases reported visiting a petting zoo and contact with a reptile more. There were significant variations by Salmonella subtype in both foods and exposures. We recommend departments conduct this analysis to generate a baseline comparison and a running average of relevant odds ratios allowing staff to focus on trace-back of contaminated food items earlier in the outbreak investigation process.
\end{abstract}

\section{Introduction}

Salmonella and Campylobacter are the top two leading causes of bacterial foodborne diarrhoeal illness in the USA causing an estimated 1000000 and 850000 cases of illness every year, respectively [1]. Salmonella is most common in children and is transmitted through contaminated food and water or contact with infected animals, typically birds and reptiles [2]. Campylobacter, one of the top causative agents for travellers' diarrhoea [3], is more common in children under 5-years-old and young adults [4]. Infection with Campylobacter is often associated with handling or consuming raw or undercooked poultry, contaminated water, or coming into contact with infected animals. In addition, consuming contaminated produce, contact with puppies and recent foreign travel are potential risk factors [5]. While both pathogens create a significant public health burden, Salmonella is more often found to be the cause of large-scale foodborne outbreaks. In 2015, 902 foodborne disease outbreaks were reported, resulting in 15202 illnesses, 950 hospitalisations, 15 deaths and 20 food recalls [6]. Campylobacter cases are more often reported as sporadic cases [7]. These differences in exposures and presentation allow for the use of interview data from patients with campylobacteriosis to act as comparison cases or 'controls' for routine surveillance monitoring and outbreak investigations.

Case-case methods are becoming a more routine practice within health departments to generate hypotheses during outbreaks $[8,9]$. This study design is similar to a case-control, but instead of non-ill controls, comparison cases are used, often from another pathogen [10-12]. These studies use odds ratios (ORs) and logistic regression, with the exposures in one set of cases compared with exposures in another set of cases. While they do not offer the measure of risk provided through case-control or cohort studies, if utilised and interpreted appropriately, they can be an important disease investigation tool $[11,13]$. These designs are useful in large part because the data utilised are often already collected through routine surveillance activities. Besides reduced time and effort, other benefits of case-case methods include decreased recall and selection bias [14]. Without having to contact and interview nonill controls, analyses can move forward quickly and with fewer staff resources [12]. The most common use of these studies within health departments has been for outbreaks of enteric diseases. 
The benefit of using Campylobacter as the comparison cases or 'controls' for these types of studies is that (1) there are generally many cases in a given jurisdiction as Campylobacter is one of the top enteric diseases reported and (2) outbreaks of Campylobacter are rare so that cases within a surveillance system will generally represent the risk factors of routine cases with very few unusual or rare exposures. This has been demonstrated with other studies that have used Campylobacter as a comparison against several other enteric diseases as well as different serotypes of Salmonella [10].

The primary objective of this analysis is to demonstrate how case-case methods can be used for routine tracking of diseases captured through public health surveillance systems and how these results are best interpreted. A secondary objective is to determine the impact of outbreak cases when they are combined with routine cases in these analyses. We use real data to demonstrate these approaches.

\section{Methods}

A case-case analysis of Salmonella and Campylobacter cases was conducted with Campylobacter cases acting as the comparison group. The source population in Maricopa County, AZ, USA of over 4 million residents in central Arizona is served by the Maricopa County Department of Public Health (MCDPH) [15]. The county encompasses both urban and rural settings and is ethnically diverse with approximately $30 \%$ of the population reported to be Hispanic [15]. Once a patient has a laboratory confirmed positive test, the county health department is notified, as required by state law, and then attempts to contact the patient for an interview. Exclusions include residency in another county, language other than English or Spanish, or current incarceration. These interviews are conducted in English and Spanish by the MCDPH staff and graduate students at the University of Arizona's College of Public Health. Questions are related to demographics, symptoms, duration, travel and food, water and animal exposures. Patients are also asked if they have any chronic conditions. This is based on self-assessment, but specific conditions are asked including diabetes, irritable bowel syndrome (IBS), gastritis and arthritis. Interview data for each pathogen collected from 2012 to 2015 were merged based on common variables and questions to create a final dataset of 2980 cases. Follow-up was slightly higher for Salmonella cases compared with Campylobacter cases (80\% and 66\%, respectively) mainly due to prioritisation of interviews.

Descriptive statistics comparing Salmonella cases to Campylobacter cases were computed and chi-square tests were used to test for differences. Unadjusted and adjusted logistic regression models were used to estimate ORs for single exposures and symptoms between Salmonella and Campylobacter cases. Salmonella cases were coded as ' 1 ', so that ORs greater than one are interpreted as the odds of exposure being greater for Salmonella cases compared with Campylobacter cases. Odds ratios under 1.0 are interpreted as an increased odds of exposure for Campylobacter cases as compared with Salmonella cases. Adjusted regression models controlling for gender, ethnicity (Hispanic, non-Hispanic) and age category were used. Age was categorised into the following groups: less than 1 year, 1-4 years, 5-9 years, 10-19 years, 20-64 years and 65 years and over. Forty-three unadjusted and 43 adjusted models comparing the odds of Salmonella to Campylobacter were fit. Data were complete for all variables with the exception of the location of residence because the question was not added to the Salmonella questionnaire until 2014. Analysis of data available showed no differences by pathogen and this variable was excluded from all regression analyses.

Salmonella Enteritidis $(n=262)$, Typhimurium $(n=176)$ and Poona $(n=151)$ were also examined by serotype. Adjusted logistic regression models were fit separately for each of these three serotypes to compare exposures and symptoms against Campylobacter cases (all Campylobacter serotypes were included, but the vast majority are Campylobacter jejuni, this is why subtype analyses of Campylobacter could not be conducted). The Wald Chi-square statistic was used to assess the significance and 95\% confidence intervals (CIs).

Finally, a sensitivity analysis was conducted to examine the effects that excluding Salmonella outbreak cases had on the measures of association. Adjusted models were constructed excluding any Salmonella cases associated with a known outbreak $(n=477)$, leaving a nested dataset of 2503 cases. Outbreak cases due to Campylobacter made up less than $7.9 \%$ of the total dataset and were included for both analyses. Results from the full and nested analyses were then compared and reviewed for differences. All analyses were performed using SAS version 9.4 (Cary, NC).

\section{Results}

A total of 2980 patients were included in the analyses, 1027 Campylobacter cases and 1953 Salmonella cases (Table 1). Salmonella cases tended to be younger with $43.6 \%$ of these cases less than 20-years-old compared with only $30.5 \%$ for Campylobacter cases. There was a difference by ethnicity, with a higher proportion of Campylobacter cases reporting Hispanic ethnicity than Salmonella cases $(P=0.01)$. Gender and race were also significantly different between groups with Campylobacter cases having a higher proportion of females (53\% to $44 \%$, $P<0.0001)$ and more likely to be White $(87 \%$ to $83 \%, P<0.0001)$ than Salmonella cases (depending on their origin, many Hispanics in Arizona would identify as White or 'Other' for race and Hispanic for ethnicity).

Symptoms and other health-related risk factors were as expected for the two diseases but had notable differences when comparing the two (Table 2). Almost all cases reported diarrhoea (98\% for Campylobacter and 92\% for Salmonella), with the majority also reporting abdominal pain, fever and nausea. A larger proportion of Salmonella cases were hospitalised (43\%) as compared with Campylobacter cases (27\%). Campylobacter cases were more likely to use antacids in the month prior to illness onset ( $21 \%$ to $16 \%, P=0.01)$. No difference was observed in antibiotic use prior to illness for $11 \%$ and $12 \%$ of Campylobacter and Salmonella cases, respectively $(P=0.61)$. Thirty-four percent of Campylobacter cases reported having a chronic condition such as diabetes, IBS, or gastritis. For Salmonella cases, the majority of responses were missing because this question was not included until 2015, however, of the 153 people who responded, $26 \%$ reported having a pre-existing condition.

For the multivariable regression models (Table 3), the odds of experiencing diarrhoea was higher among Campylobacter patients $(\mathrm{OR}=0.21)$, whereas odds of experiencing nausea $(\mathrm{OR}=1.39)$ and vomiting $(\mathrm{OR}=1.79)$ were higher among Salmonella cases. The odds of seeking healthcare provided through an urgent care or ER were higher among Salmonella cases $(\mathrm{OR}=2.10)$ and the odds of hospitalisation were also 3.5 times higher for Salmonella cases compared with Campylobacter cases. By Salmonella serotype, 
Table 1. Participant characteristics by pathogen infection

\begin{tabular}{|c|c|c|c|}
\hline Characteristic & $\begin{array}{c}\text { Campylobacter } \\
\quad N=1,027\end{array}$ & $\begin{array}{c}\text { Salmonella } \\
\qquad N=1,953\end{array}$ & $P$-value \\
\hline Age & & & $<0.0001$ \\
\hline$<1$ & $18(3.0)$ & $186(9.6)$ & \\
\hline $1-4$ & $74(12.1)$ & $231(11.9)$ & \\
\hline $5-9$ & $36(5.9)$ & $184(9.5)$ & \\
\hline $10-19$ & $58(9.5)$ & $244(12.6)$ & \\
\hline $20-64$ & $338(55.4)$ & $832(43.0)$ & \\
\hline $65+$ & $86(14.1)$ & $260(13.4)$ & \\
\hline Gender & & & $<0.0001$ \\
\hline Male & $467(47.2)$ & $1097(56.5)$ & \\
\hline Female & $523(52.8)$ & $845(43.5)$ & \\
\hline Race & & & $<0.0001$ \\
\hline White & $805(87.3)$ & $1338(82.8)$ & \\
\hline African American & $25(2.7)$ & $62(3.8)$ & \\
\hline Asian & $17(1.8)$ & $32(2.0)$ & \\
\hline $\begin{array}{l}\text { American Indian/ } \\
\text { Alaska Native }\end{array}$ & $19(2.1)$ & $106(6.6)$ & \\
\hline Other & $56(6.1)$ & $78(4.8)$ & \\
\hline Ethnicity & & & 0.01 \\
\hline Non-Hispanic & $589(63.0)$ & $1127(70.1)$ & \\
\hline Hispanic & $346(37.0)$ & $480(29.9)$ & \\
\hline
\end{tabular}

Note-Race 'Hawaiian' has been grouped with other. There were 2 and 0 observations.

the odds of symptoms including fever was higher for S. Typhimurium $(\mathrm{OR}=1.83)$ and $S$. Poona $(\mathrm{OR}=2.12)$ compared with Salmonella overall and the odds of experiencing vomiting was higher for $S$. Enteritidis $(\mathrm{OR}=2.05)$ and S. Typhimurium $(\mathrm{OR}=2.11)$ compared with Salmonella overall.

ORs for a given exposure that were statistically significantly less than 1.0 (i.e., had higher odds of those exposures among patients infected with Campylobacter as compared to Salmonella) included peppers $(\mathrm{OR}=0.52)$, sprouts $(\mathrm{OR}=0.60)$, fruit other than cantaloupe $(\mathrm{OR}=0.76)$, queso fresco $(\mathrm{OR}=$ 0.57) (a Mexican-style white cheese that is often sold unpasteurised), poultry $(\mathrm{OR}=0.60)$, eggs $(\mathrm{OR}=0.22)$, raw juice $(\mathrm{OR}=$ $0.46)$, raw dairy $(\mathrm{OR}=0.47)$, raw nuts $(\mathrm{OR}=0.55)$ and peanut butter $(\mathrm{OR}=0.72)$. Campylobacter case-patients also had higher odds of reporting a variety of animal exposures including birds $(\mathrm{OR}=0.45)$, pets $(\mathrm{OR}=0.52)$, sick pets $(\mathrm{OR}=0.27)$, dairy animals $(\mathrm{OR}=0.48)$ and contact with animal products $(\mathrm{OR}=0.52)$ including hides and manure. The two exceptions to animal exposures were reptiles $(\mathrm{OR}=1.77)$ and petting zoos $(\mathrm{OR}=$ 4.35 ) indicating that Salmonella cases had higher odds of reporting these exposures.

The analyses by serotype were consistent overall with the results for all Salmonella cases combined. Some notable exceptions were travel to Mexico, where the overall odds ratio was $1.20(P=0.38)$ whereas the odds of being a S. Enteritidis case compared with Campylobacter had an odds ratio of $3.28(95 \%$ CI: 1.8-6.0). The odds of reptile exposure were higher among cases of S. Typhimurium $(\mathrm{OR}=2.30)$ and $\mathrm{S}$. Poona $(\mathrm{OR}=2.73)$ than Salmonella overall $(\mathrm{OR}=1.77)$.
Table 2. Symptoms and exposures by pathogen

\begin{tabular}{|c|c|c|c|}
\hline Symptoms \& Exposures & $\begin{array}{c}\text { Campylobacter } \\
N(\%)\end{array}$ & $\begin{array}{c}\text { Salmonella } \\
\text { N (\%) }\end{array}$ & $P$-value \\
\hline Diarrhoea & 969 (98.1) & $1228(91.5)$ & $<0.0001$ \\
\hline Fever & $658(72.2)$ & $922(74.1)$ & 0.32 \\
\hline Nausea & $582(61.1)$ & $798(65.3)$ & 0.04 \\
\hline Vomit & $374(37.7)$ & $615(48.7)$ & $<0.0001$ \\
\hline Abdominal Pain & $797(83.5)$ & $965(81.3)$ & 0.19 \\
\hline Headache & $535(58.7)$ & $634(60.8)$ & 0.35 \\
\hline $\begin{array}{l}\text { Antibiotics in month } \\
\text { prior }\end{array}$ & $108(11.1)$ & $127(11.8)$ & 0.61 \\
\hline Antacids in month prior & $204(21.2)$ & $170(16.1)$ & 0.01 \\
\hline Medical Care & & & $<0.0001$ \\
\hline Primary Care & $476(47.9)$ & $298(24.8)$ & \\
\hline Hospitalisation & $264(26.6)$ & $515(42.9)$ & \\
\hline ED/Urgent Care Only & $254(25.6)$ & $387(32.3)$ & \\
\hline Chronic condition & & & 0.01 \\
\hline Yes & $337(34.3)$ & $259(26.4)$ & \\
\hline No & $645(65.7)$ & $722(73.6)$ & \\
\hline
\end{tabular}

Finally, the sensitivity analysis showed very few differences when the outbreak cases $(n=477)$ were excluded. There was no consistent change in direction, with some odds ratios increasing when outbreak cases were excluded and others decreasing. For those odds ratios that did change, the change was less than $10 \%$ for all risk factors with the exception of eggs which changed $12 \%$ and raw nuts $(11 \%)$. The odds of reporting exposure to a river, lake, or pond were $27 \%$ higher among Campylobacter cases when Salmonella outbreak cases were excluded.

\section{Discussion}

In order to effectively use these methods to identify a potential cause of an outbreak or track changes in disease trends over time, it is necessary for health agencies to establish baseline levels to determine how these diseases compare with one another under 'normal' circumstances and what cases may be appropriate to include or exclude from the analysis. Deviations from these baseline odds ratios may yield clues for identifying the source of an outbreak or even tracking changes in exposures. For this study, we used 4 years of data that was available based on survey implementation. We recommend departments conduct this analysis on a yearly basis and when the data permits to create a 5 year running average of relevant odds ratios for comparison. Similar to how other clusters are identified using 2 standard deviations above a 5 year mean by month for surveillance data, by monitoring this data on a regular basis, clusters or temporal changes in risk factors may be identified earlier.

Using this data, the health department can quickly and easily compare data from suspected Salmonella outbreak cases to Campylobacter cases and compare these values to their baseline. For an outbreak tied to a specific risk factor, the odds ratio would be expected to increase from the baseline value. The percent change would be based on the percent exposed in the outbreak cases, sample size and specific risk factor under 
Table 3. Adjusted logistic regression models of symptoms and risk factors

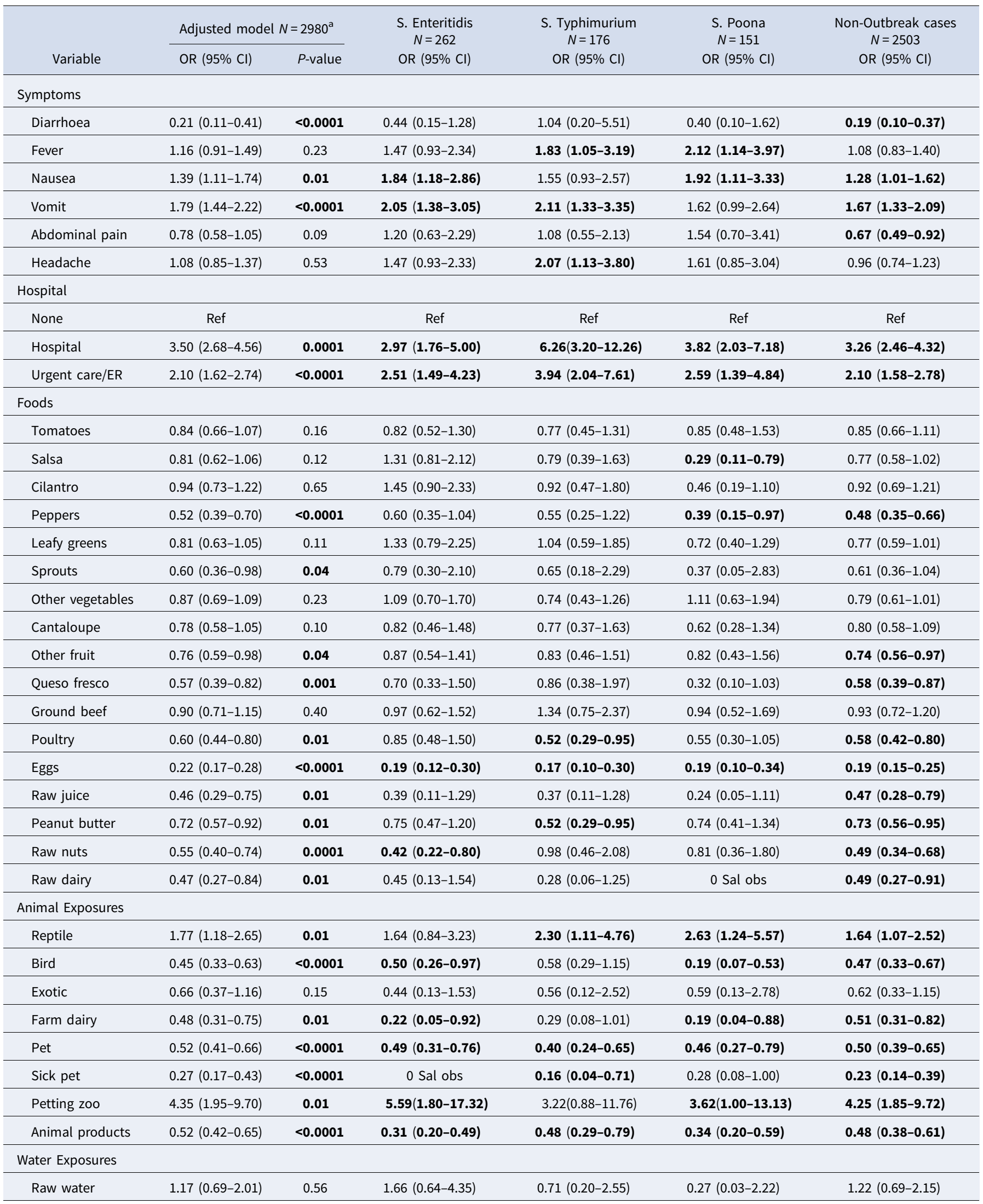


Table 3. (Continued.)

\begin{tabular}{|c|c|c|c|c|c|c|}
\hline \multirow[b]{2}{*}{ Variable } & \multicolumn{2}{|c|}{ Adjusted model $N=2980^{a}$} & \multirow{2}{*}{$\begin{array}{l}\text { S. Enteritidis } \\
\quad N=262 \\
\text { OR }(95 \% \mathrm{Cl})\end{array}$} & \multirow{2}{*}{$\begin{array}{l}\text { S. Typhimurium } \\
\qquad N=176 \\
\text { OR }(95 \% \mathrm{Cl})\end{array}$} & \multirow{2}{*}{$\begin{array}{c}\text { S. Poona } \\
N=151 \\
\text { OR }(95 \% \mathrm{Cl})\end{array}$} & \multirow{2}{*}{$\begin{array}{c}\text { Non-Outbreak cases } \\
\qquad N=2503 \\
\text { OR }(95 \% \mathrm{Cl})\end{array}$} \\
\hline & OR $(95 \% \mathrm{Cl})$ & $P$-value & & & & \\
\hline \multicolumn{7}{|l|}{ Swim } \\
\hline None & Ref & & Ref & Ref & Ref & Ref \\
\hline River, lake, pond & $0.24(0.08-0.71)$ & 0.01 & 0 Sal obs & 0 Sal obs & 0 Sal obs & $0.18(0.04-0.76)$ \\
\hline Pool, hot tub & $1.03(0.69-1.53)$ & 0.90 & 0 Sal obs & 0 Sal obs & 0 Sal obs & $0.80(0.49-1.31)$ \\
\hline \multicolumn{7}{|l|}{ Other } \\
\hline Mexico & $1.20(0.80-1.78)$ & 0.38 & $3.28(1.80-6.00)$ & $0.68(0.22-2.06)$ & $0.21(0.03-1.61)$ & $0.94(0.60-1.46)$ \\
\hline Chronic & $0.78(0.60-0.99)$ & 0.05 & $0.38(0.21-0.66)$ & $0.51(0.24-1.05)$ & $0.67(0.30-1.47)$ & $0.74(0.56-0.96)$ \\
\hline Antibiotics before & $1.22(0.86-1.72)$ & 0.27 & $0.55(0.24-1.26)$ & $0.85(0.35-2.05)$ & $0.81(0.34-1.92)$ & $1.37(0.96-1.97)$ \\
\hline Antacid before & $0.69(0.52-0.90)$ & 0.01 & $0.41(0.22-0.77)$ & $1.13(0.53-2.39)$ & $0.70(0.30-1.60)$ & $0.70(0.52-0.94)$ \\
\hline \multicolumn{7}{|l|}{ Raw handle } \\
\hline None & Ref & & Ref & Ref & Ref & Ref \\
\hline Ground beef & $0.78(0.39-1.60)$ & 0.50 & 0 Sal obs & 0 Sal obs & 0 Sal obs & $0.60(0.25-1.42)$ \\
\hline Poultry & $0.93(0.57-1.51)$ & 0.75 & 0 Sal obs & 0 Sal obs & 0 Sal obs & $0.77(0.44-1.36)$ \\
\hline Both & $0.55(0.34-0.89)$ & 0.02 & 0 Sal obs & 0 Sal obs & 0 Sal obs & $0.48(0.27-0.85)$ \\
\hline
\end{tabular}

ORs > 1 indicate exposure was more likely in Salmonella cases than Campylobacter cases; OR $<1$ indicate exposure was more likely in Campylobacter cases than Salmonella cases; 0 Sal obs, zero exposures for the variable among all salmonella cases. Bolded values indicate statistically significant results at the 0.05 level.

${ }^{a}$ Models adjusted for age category (see Table 1 for each category), gender and ethnicity.

investigation. For known outbreaks, there is not yet a specific absolute change (e.g. a 25\% difference from baseline for outbreak cases) that can be suggested to identify a cause. However, as these methods are utilised more broadly and reported, a range can be determined. For future work, modelling can be used to simulate outbreaks to determine the sample size and measure of effect necessary to properly identify a causative agent.

Overall, our results were similar to what is currently known about these two diseases for both symptoms and risk factors. The majority of exposures were reported more frequently by patients with Campylobacter compared with Salmonella. This may indicate that in Arizona, exposure to Campylobacter is more wide-spread across a variety of food exposures, again, making these cases a good choice as 'controls' compared with a variety of enteric pathogens. Foods such as poultry and queso fresco, which previous studies in the state have shown to be associated with Campylobacter [16], are also more likely to be reported as such here. Eggs, often associated with Salmonella, were not associated in this dataset, even when examined by serotype. For Salmonella, the two most striking results were related to animal exposures. The odds of reporting both reptiles $(\mathrm{OR}=1.77)$ and petting zoos $(\mathrm{OR}=4.35)$ were much higher among patients with salmonellosis and is consistent with a meta-analysis of risk factors by Domingues, et al. [2]. While known pre-disposing risk factors such as antibiotic use or antacid use in the prior month [17] were not different by a pathogen, there were statistical differences in reported chronic conditions, with significantly more Campylobacter patients reporting a pre-existing condition. This same relationship held true after adjustment but was only barely significant, likely due to the higher numbers of young children with Salmonella.

The three Salmonella serotypes, Enteritidis, Typhimurium and Poona were selected because the first two are the most common serotypes seen nationally [18] and S. Poona was selected because it is an emerging serotype responsible for a large national outbreak associated with cucumbers in 2015, with 140 reported cases in Arizona [19,20]. Generally, the odds ratios were similar by serotype when compared with Campylobacter cases and to the full Salmonella dataset. S. Enteritidis was more often associated with petting zoos and travel to Mexico. The odds ratios for S. Poona were lower than the odds ratio for the full Salmonella dataset for most exposures except reptile exposure $(\mathrm{OR}=2.73)$, a known risk factor [21]. The selection of these serotypes was used to demonstrate that differences in risk factor profiles can be identified using these methods and may be useful in an outbreak where the serotype has been identified.

The sensitivity analysis compared the full dataset (all Salmonella cases included) to a subset where any Salmonella cases associated with a known outbreak were excluded. The results show very little differences between the two analyses indicating that it is likely unnecessary to exclude these cases for this type of analysis allowing for a larger sample size and greater power. In addition, there is no misclassification bias due to including cases that were, in fact, part of an outbreak, but not identified as such for a variety of reasons. The similarities are not surprising given that outbreak cases make up a minority of cases and are spread across a wide variety of causal factors. However, it is important for other jurisdictions utilising these methods to conduct their own sensitivity analysis and decide the best course of action based on their local information.

Like all studies that utilise surveillance data there are strengths and limitations. Surveillance data are often under-utilised and an excellent potential source of public health information. In addition, when working with a highly efficient surveillance system such as the one in $\mathrm{MCDPH}$, with high rates of follow-up and timely interviews, issues such as recall and selection bias are of less concern. Another strength was the study population. Maricopa County represents a large ethnically diverse area that consists of both urban and rural areas allowing these results to be more generalisable in other areas in this region of the USA. 
One key element of this project was the mapping of questions and variables. From 2014 to present, all Campylobacter and Salmonella cases were interviewed using the same standardised questionnaire. For data collected in 2012-2013, questions and variables were mapped to current questions to ensure information was accurately linked. While this data could have been excluded, it is important to demonstrate that questions on different surveillance forms can be mapped to one another for comparative analysis. Variables that were not asked consistently during this time period or had a high volume of missing values from one or both pathogens were excluded. This limits the comparisons that can be made. It is the recommendation of the authors that jurisdictions who are interested in utilising case-case methods carefully review surveillance forms and standardise the language where possible to increase the number of variables that can be included. Due to changes in case definitions, we only used culture-confirmed cases to reduce any chance of misclassification bias.

These methods and information are also in-line with the Center for Disease Control and Prevention's (CDC) Case Ascertainment project to collect and map questionnaires for several enteric diseases, including Salmonella and Campylobacter from select sites around the country [22]. These types of analyses also put a large amount of routine surveillance data health departments regularly collect to use beyond its mandated need for reporting. This study can provide health departments a tool to develop baseline values that will then allow for quick and easy comparison of surveillance data for routine analysis of risk factors and of outbreak cases.

Acknowledgements. The authors would like to thank the students of SAFER and the disease investigators of Maricopa County Department of Public Health for conducting interviews and Lindsay Kohler and Amit Arora who helped with data cleaning and merging over the years.

Conflict of interest. None.

\section{References}

1. Scallan E et al. (2011) Foodborne illness acquired in the United States-Major pathogens. Emerging Infectious Diseases 17, 7-15.

2. Domingues AR et al. (2012) Source attribution of human salmonellosis using a meta-analysis of case-control studies of sporadic infectionssystematic review. Epidemiology and Infection 140, 959-969.

3. Brunette G (2017) Chapter 3 Infectious diseases related to travel: Campylobacteriosis. In Chief E et al. (eds). CDC Yellow Book 2018: Health Information for International Travel. Atlanta: Centers for Disease Control and Prevention. Available at https://wwwnc.cdc.gov/travel/yellowbook/ 2018/infectious-diseases-related-to-travel/campylobacteriosis

4. CDC (2014) CDC Food Safety-Campylobacter. June 3.

5. Domingues AR et al. (2012) Source attribution of human campylobacteriosis using a meta-analysis of case-control studies of sporadic infections. Epidemiology and Infection 140, 970-981.
6. CDC (2017) Surveillance for Foodborne Outbreaks United States, 2015 : Annual Report. Atlanta, GA.

7. CDC FDASN (FoodNet) (2014) 2014 FoodNet Annual Surveillance Report (Final Report). Atlanta, GA.

8. Gaulin C et al. (2012) Escherichia coli O157:H7 outbreak linked to raw milk cheese in Quebec, Canada: use of exact probability calculation and casecase study approaches to foodborne outbreak investigation. Journal of Food Protection 75, 812-818.

9. Krumkamp R, Reintjes R and Dirksen-Fischer M (2008) Case-case study of a Salmonella outbreak: an epidemiologic method to analyse surveillance data. International Journal of Hygiene and Environmental Health 211, 163-167.

10. Aiken AM, Lane C and Adak GK (2010) Risk of Salmonella infection with exposure to reptiles in England, 2004-2007. Euro Surveillance: European Communicable Disease Bulletin 15, 19581.

11. Gillespie IA et al. (2002) A case-case comparison of Campylobacter coli and Campylobacter jejuni infection: a tool for generating hypotheses. Emerging Infectious Diseases 8, 937-942.

12. Wilson $\mathbf{N}$ et al. (2008) Case-case analysis of enteric diseases with routine surveillance data: potential use and example results. Epidemiologic Perspectives \& Innovations 5, 6.

13. Pogreba-Brown K, Ernst K and Harris RB (2014) Case-case methods for studying enteric diseases: a review and approach for standardization. $O A$ Epidemiology 7, 1-9.

14. McCarthy N and Giesecke J (1999) Case-case comparisons to study causation of common infectious diseases. International Journal of Epidemiology 28, 764-768.

15. U.S. Census Bureau QuickFacts selected: Maricopa County, Arizona. Available at https://www.census.gov/quickfacts/fact/table/maricopacountyarizona/ INC110215 (Accessed 21 August 2017).

16. Pogreba-Brown K et al. (2015) Assessing risk factors of sporadic Campylobacter infection: a case-control study in Arizona. Epidemiology and Infection 144, 829-839.

17. Neal KR and Slack RC (1997) Diabetes mellitus, anti-secretory drugs and other risk factors for campylobacter gastro-enteritis in adults: a casecontrol study. Epidemiology and Infection 119, 307-311.

18. CDC (2017) Foodborne Diseases Active Surveillance Network (FoodNet): FoodNet 2015 Surveillance Report (Final Data). Atlanta, Georgia: U.S. Department of Health and Human Services, CDC.

19. CDC (2015) Case Count Maps, Multistate Outbreak of Salmonella Poona Infections Linked to Imported Cucumbers. Available at https://www.cdc. gov/salmonella/poona-09-15/map.html (Accessed 21 August 2017).

20. CDC (2015) Multistate Outbreak of Salmonella Poona Infections Linked to Imported Cucumbers (Final Update), Multistate Outbreak of Salmonella Poona Infections Linked to Imported Cucumbers. Available at https:// www.cdc.gov/salmonella/poona-09-15/index.html (Accessed 21 August 2017).

21. CDC (2013) An Atlas of Salmonella in the United States, 1968-2011: Laboratory-Based Enteric Disease Surveillance. Atlanta, Georgia: US Department of Health and Human Services, CDC.

22. Marder E (2015) Routine Collection of Exposure Data From Sporadic Salmonellosis Cases - Foodborne Diseases Active Surveillance Network, 2014. Boston, MA: CSTE. 